\title{
Multidisciplinary treatment including systemic chemotherapy for a malignant phyllodes tumour of the prostate
}

\author{
Yasukiyo Murakami, MD; ${ }^{*}$ Ken-ichi Tabata, MD; ${ }^{*}$ Atsushi Sugita, MD;" Kohei Mochizuki, MD; ${ }^{*}$ \\ Ryota Maeyama, MD,* Miyoko Okazaki, PhC;; Morihiro Nishi, MD; ${ }_{;}^{*}$ Kazumasa Matsumoto, MD; ${ }^{*}$ \\ Tetsuo Fujita, MD; Takefumi Satoh, MD; ${ }^{*}$ Shi-Xu Jiang, MD, ${ }^{\xi}$ Makoto Saegusa, MD; Masatsugu Iwamura, MD*
}

*Department of Urology, Kitasato University School of Medicine, Japan; 'Department of Pharmacology, Kitasato University School of Medicine, Japan; §Department of Pathology, Kitasato University School of Medicine, Japan

Cite as: Can Urol Assoc J 2014;8(3-4):e263-5. http://dx.doi.org/10.5489/cuaj.1648 Published online April 14, 2014.

\section{Abstract}

A 22-year-old man was referred to our hospital with macroscopic hematuria and consistent anal pain. Magnetic resonance imaging revealed an enlarged prostate tumour invading the bladder and rectum. A biopsy revealed an unclassified spindle cell sarcoma. Subsequently, radical cystoprostatectomy and resection of the rectum were performed. A histopathological examination revealed a prostatic malignant phyllodes tumour with a negative surgical margin. However, a local recurrence was identified 2 months after surgery. Induction therapy included 4 cycles of systemic chemotherapy comprising etoposide with ifosfamide and cisplatin. Although a partial response was observed at the local site, lung metastasis developed. Second-line chemotherapy with ifosfamide and doxorubicin with radiotherapy to the pelvis was administered and led to complete regression; however, its efficacy was transient. Although additional chemotherapy was administered, the patient eventually died due to the rapidly growing, recurrent tumour.

\section{Introduction}

We report a case of a malignant phyllodes tumour of the prostate, an extremely rare neoplasm, which was treated with surgery, radiation, and multiple chemotherapy regimens. We also discuss therapeutic aspects of this uncommon tumour, with a review of the literature.

\section{Case report}

A 22-year-old man was referred to our hospital because of gross hematuria and consistent anal pain. A magnetic resonance imaging showed a lobulated prostate tumour measuring $7 \mathrm{~cm}$, invading the urinary bladder and rectum. Neither lymph node nor distant metastasis was detected using systemic computed tomography (CT) and bone scintigraphy.
Laboratory tests were within normal limits, including serum prostate-specific antigen (PSA). A colonoscopy revealed a tumour within the anterior rectal wall, which indicated rectal invasion by the prostate tumour. A transperineal needle biopsy of the prostate was performed, following which a pathological diagnosis of unclassified spindle cell sarcoma was made.

We then performed a total cystoprostatectomy with continent ileal pouch, an anterior resection of the rectum with colostomy, and pelvic lymph node dissection. Microscopic findings indicated variable cellular spindle stroma with a high mitotic rate and epithelial components, which projected leaf-like stromal proliferations lined by epithelial cells. Tumour invasion of the adjacent urinary bladder and rectal wall was confirmed. There was no evidence of lymph node metastasis, and the surgical margin was negative (Fig.1a, Fig.1b).

Immunohistochemical analysis showed that the spindle stromal cells were positive for CD10 and vimentin, but negative for PSA, smooth muscle antigen, p53, desmin, CD34, S-100, androgen receptor, estrogen receptor, and cytokeratins AE1/AE3. The percentage of mindbomb homolog-1positive cells among the stromal cells was $60 \%$, whereas epithelial membrane antigen, AE1/AE3, and prostatic acid phosphatase were positive in the columnar cell lining the epithelial components, leading to a diagnosis of a high-grade malignant phyllodes tumour of the prostate.

Two months after the surgery, a CT scan revealed local recurrence in the pelvis and bilateral external iliac lymph nodes (Fig. 2a). For induction therapy, we treated the patient with VIP chemotherapy (etoposide, $80 \mathrm{mg} / \mathrm{m}^{2}$; ifosfamide, $1200 \mathrm{mg} / \mathrm{m}^{2}$; and cisplatin, $20 \mathrm{mg} / \mathrm{m}^{2}$ on days 1 to 5$)$. Because a good response was achieved, 4 cycles of chemotherapy were administered. However, after the chemotherapy, an additional CT scan revealed a $1-\mathrm{cm}$ metastatic tumour in the patient's lung. The patient then received a second-line treatment consisting of 6 cycles of Al chemotherapy (ifosfamide, $2000 \mathrm{mg} / \mathrm{m}^{2}$ on day 1 and doxorubicin, $30 \mathrm{mg} / \mathrm{m}^{2}$ on 


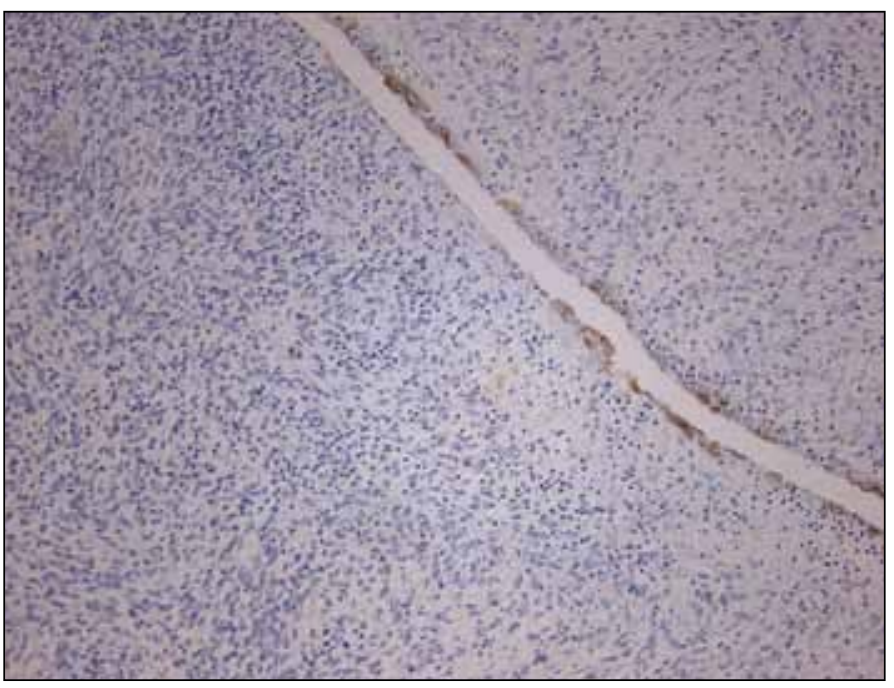

Fig. 1a. Papanicolaou (PAP) staining of a phyllodes tumour of the prostate containing slit-like epithelium-lined spaces and variable cellular spindle cell stroma. Epithelial cells were PAP-positive, but stromal cells were PAP-negative (magnification $\times 40$ )

days 1 to 3) with 50 Gy of radiotherapy to the entire pelvis. Following these therapies, a CT scan indicated complete regression of the local recurrence and lung metastasis (Fig. $2 b)$.

However, a second local recurrence in the pelvis with right ureter involvement was diagnosed after 3 months. Although 2 additional cycles of Al chemotherapy were administered, the $\mathrm{CT}$ revealed progressive disease. For the third-line chemotherapy, the patient was treated with TIN chemotherapy (ifosfamide, $2000 \mathrm{mg} / \mathrm{m}^{2}$ on days $1-3$; paclitaxel, $175 \mathrm{mg} / \mathrm{m}^{2}$; and nedaplatin, $100 \mathrm{mg} / \mathrm{m}^{2}$ on day 1 ). Despite 2 cycles of TIN chemotherapy, the patient died because of the rapidly growing tumour 20 months after the surgery.

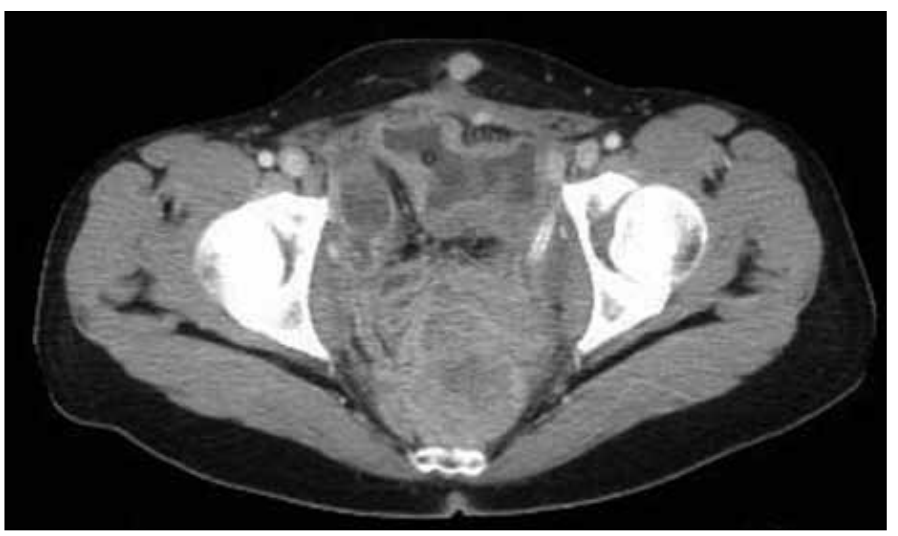

Fig. 2a. Pelvic computer tomography scan at the time of recurrence.

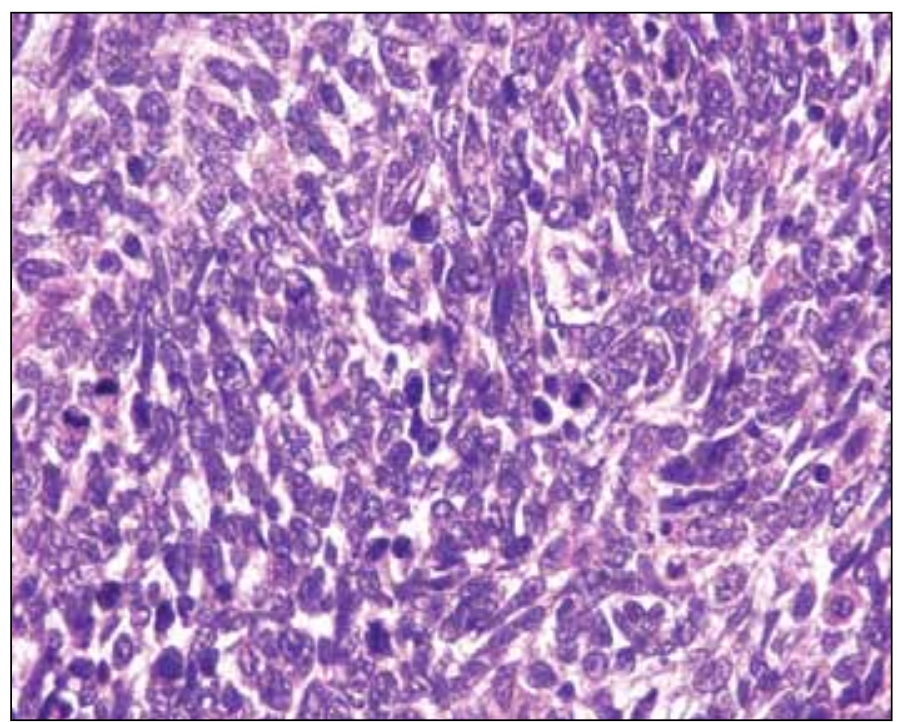

Fig. $1 \boldsymbol{b}$. Proliferation of elongated and spindle-shaped cells within the stroma (hematoxylin and eosin staining; original magnification $\times 400$ ).

\section{Discussion}

Phyllodes tumour of the prostate is an extremely rare neoplasm. To our knowledge, 85 cases of phyllodes tumours of the prostate have been reported. Histologically, a phyllodes tumour of the prostate is characterized by a distinctive biphasic pattern with hyperplastic epithelium-lined cysts, leaf-like intraluminal and epithelium-lined stromal projections, compressed and elongated slit-like epithelium-lined spaces, and variable cellular spindle cell stromas. ${ }^{1}$ Phyllodes tumour is histologically classified into benign, border-line, or malignant depending on the mitotic activity, stromal cellularity, and stromal/epithelial ratio. ${ }^{2}$ However, previous reports have suggested that the histological appearance does not always correlate with the clinical behaviour of this neoplasm. ${ }^{1}$

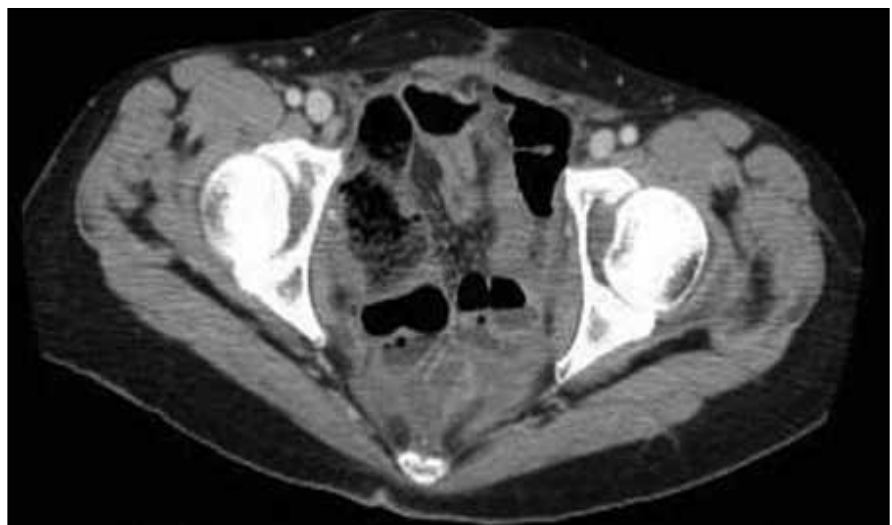

Fig. $\mathbf{2 b}$. Pelvic computer tomography scan after chemoradiotherapy showing near complete regression of the tumour. 
Radical surgery is the treatment of choice for malignant phyllodes tumour of the prostate. The efficacy of radiotherapy and chemotherapy for a phyllodes tumour has not been well-established because of the rarity of these masses, although some reports have shown their efficacy.

Although 3 cases of phyllodes tumours treated with chemotherapy have been reported, ${ }^{3-5}$ the present case is the first to document the outcomes of multiple chemotherapy regimens (VP-16, ifosfamide, cisplatin $\rightarrow$ doxorubicin, ifosfamide $\rightarrow$ ifosfamide, paclitaxel, and nedaplatin).

Sakura and colleagues ${ }^{3}$ reported the efficacy of VIP chemotherapy for lymph node metastasis after prostatectomy in a 19-year-old male with a prostatic malignant phyllodes tumour. Similarly, we chose VIP as the first-line chemotherapy in our patient because of the corresponding clinical characteristics, including age and metastatic site, and because we wished to achieve complete regression. Then, we switched to the $\mathrm{Al}$ regimen (doxorubicin and ifosfamide) because the tumour metastasized to the patient's lung. Lam and colleagues reported the efficacy of ifosfamide and doxorubicin for lung metastasis following radical prostatectomy and adjuvant radiotherapy for malignant phyllodes tumours of the prostate. ${ }^{4}$ These regimens are also recognized as key treatments for soft tissue sarcomas. Therefore, we chose this regimen as the second-line chemotherapy. Six courses of $\mathrm{Al}$ chemotherapy and concurrent salvage radiation therapy led to near complete regression. However, its efficacy was transient.

We finally chose the TIN regimen as the third-line chemotherapy. A previous report demonstrated the efficacy of an alternative therapy of paclitaxel and docetaxel for a patient with lung metastasis from a malignant phyllodes tumour of the breast. Nonomura and colleagues found that paclitaxel enhanced drug activity and possible synergistic effects when combined with ifosfamide or nedaplatin. ${ }^{6}$ Moreover, an in vitro study demonstrated that $28 \%$ of cisplatin-resistant tumours remained sensitive to nedaplatin. ${ }^{7}$ However, TIN chemotherapy did not demonstrate any response as a thirdline treatment in our case.

\section{Conclusion}

To enhance the therapeutic efficacy of surgical treatment, which is the standard therapy for prostatic malignant phyl- lodes tumours, perioperative chemotherapy and radiotherapy may be effective. However, even in phyllodes tumours of the breast, the role of perioperative treatment has not been fully defined. ${ }^{8}$ The difficulty of preoperative diagnosis in biopsy samples also hinders neoadjuvant treatment. ${ }^{9}$ Therefore, to improve the poor prognosis of prostatic malignant phyllodes tumours, we need to consider not only treatment strategies but also diagnostic advancements.

Competing interests: Dr. Murakami, Dr. Tabata, Dr. Sugita, Dr. Mochizuki, Dr. Maeyama, Dr. Okazaki, Dr. Nishi, Dr. Matsumoto, Dr. Fujita, Dr. Satoh, Dr. Jiang, Dr. Saegusa and Dr. Iwamura all declare no competing financial or personal interests.

This paper has been peer-reviewed.

\section{References}

1. Bostwick DG, Hossain D, Qian J, et al. Phyllodes tumor of the prostate: Long-term follow-up study of 23 cases. J Urol 2004;172:894-9. http://dx.doi.org/10.1097/01.ju.0000134580.71261.57

2. Young JF, Jensen PE, Wiley CA. Malignant phyllodes tumor of the prostate: A case report with immunohistochemical and ultrastructural studies. Arch Pathol Lab Med 1992;1 16:296-9.

3. Sakura M, Tsukamoto T, Yonese J, et al. Successful therapy of a malignant phyllodes tumor of the prostate after postoperative local failure. Urology 2006;67:845.e11-3. http://dx.doi.org/10.1016/i. urology.2005.10.011

4. Lam KC, Yeo W. Chemotherapy induced complete remission in malignant phyllodes tumor of the prostate metastasizing to the lung. J Urol 2002;168:1 104-5. http://dx.doi.org/10.1097/01. ju.0000024782.25669.06

5. Agrawal V, Sharma D, Wadhwa N. Case report: Malignant phyllodes tumor of prostate. Int Urol Nephrol 2003;35:37-9.

6. Nonomura N, Oka D, Nishimura K, et al: Paclitaxel, ifosfamide, and nedaplatin (TIN) salvage chemotherapy for patients with advanced germ cell tumors. Int J Urol 2007;14:527-31. http://dx.doi.org/10.1111 /i.1442-2042.2006.01702.x

7. Alberts DS, Fanta PT, Running KL, et al. In vitro phase II comparison of the cytotoxicity of a novel platinum analog, nedaplatin (254-S), with that of cisplatin and carboplatin against fresh, human ovarian cancers. Cancer Chemother Pharmacol 1997;39:493-7.

8. Morales-Vasquez F, Gonzalez-Angulo AM, Broglio K, et al. Adjuvant chemotherapy with doxorubicin and dacarbazine has no effect in recurrence-free survival of malignant phyllodes tumors of the breast. Breast J 2007;13:551-6. http://dx.doi.org/10.1111/i.1524-4741.2007.00510.x

9. Fuji T, Shimada K, Tanaka N, et al. Phyllodes tumor of the prostate. Pathol Int 2012;62:204-8. http:// dx.doi.org/10.1111/i.1440-1827.2012.02796.x

Correspondence: Dr. Yasukiyo Murakami, Department of Urology, Kitasato University School of Medicine, Japan; yaskiyomura@yahoo.co.jp 